MAGNIMS group and in 2016 by the CMSC are important for the diagnosis and monitoring of patients with multiple sclerosis (MS) and for the appropriate use of MRI in routine clinical practice. Methods: Two panels of experts convened to update existing guidelines for a standardized MRI protocol. The MAGNIMS panel convened in Graz, Austria in April 2019. The CMSC NAIMS panel met separately and independently in Newark, USA in October 2019. Subsequently, the MAGNIMS, NAIMS, and CMSC working groups combined their efforts to reach an international consensus Results: The revised guidelines on MRI in MS merges recommendations from MAGNIMS, CMSC, and NAIMS to improve the use of MRI for diagnosis, prognosis and monitoring of individuals with MS. 3D acquisitions are emphasized for optimal comparison over time. Core brain sequences include a 3D-T2wFLAIR for lesion identification and monitoring treatment effectiveness. Gadolinium-based contrast is recommended for diagnostic studies and judicious use for routine monitoring of MS patients. DWI sequences are recommended for PML safety monitoring. Conclusions: The international consensus guidelines strive for global acceptance of a useful and usable standard of care for patients with MS.

\section{Neurocritical Care}

\section{P.050}

\section{Perspectives on the use of ancillary tests for determining neurological death: a survey of Canadian intensivists}

J Neves Briard (Montreal)* M Yu (Montreal) LP Carvalho (Montreal) SW English (Ottawa) $F$ d'Aragon (Sherbrooke) $F$ Lauzier (Quebec) A Turgeon (Quebec) $S$ Dhanani (Ottawa) $L$ McIntyre (Ottawa) SD Shemie (Montreal) G Knoll (Ottawa) DA Fergusson (Ottawa) SJ Anthony (Toronto) MJ Weiss (Quebec), M Chassé (Montreal)

doi: 10.1017/cjn.2021.331

Background: Ancillary tests are indicated to diagnose death by neurological criteria whenever clinical neurological examination is unreliable, but their use is variable and subject to debate. Methods: Survey of Canadian intensivists providing care for potential organ donors. We included closed-ended questions and different clinical scenarios regarding the use of ancillary tests. Results: Among 550 identified intensivists, 249 completed the survey. Respondents indicated they would be comfortable diagnosing death based on neurological examination without ancillary tests in the following scenarios: movement in response to stimulation (48\%), spontaneous peripheral movement $(31 \%)$, inability to evaluate upper/lower extremity responses $(34 \%)$ or both oculocephalic and oculo-caloric reflexes (17\%), presence of high cervical spinal cord injury $(16 \%)$ and within 24 hours of hypoxemic-ischemic brain injury (15\%). Furthermore, 93\% agreed that ancillary tests should always be conducted when a complete neurological examination is impossible, $89 \%$ if there remains possibility of residual sedative effect and 59\% in suspected isolated brainstem death. Conclusions: Our findings suggest that Canadian intensivists have different perceptions on what constitutes a complete and reliable clinical neurological examination for determining death by neurologic criteria. Some self-reported practices also diverge from national recommendations. Further investigation and education are required to align and standardize medical practice across physicians and systems.

\section{P.051}

Do clinical confounders to the neurological examination modify the diagnostic accuracy of CT-angiography for death by neurological criteria/brain death?

$J$ Neves Briard (Montreal)* R Nitulescu (Montreal) É Lemoine (Montreal) S English (Ottawa) L McIntyre (Ottawa) G Knoll (Ottawa) S Shemie (Montreal) C Martin (London) A Turgeon (Québec City) F Lauzier (Québec City) D Fergusson (Ottawa), M Chassé (Montreal)

doi: 10.1017/cjn.2021.332

Background: CT-angiography is an ancillary test used to diagnose death by neurological criteria (DNC), notably in cases of unreliable neurological examinations due to clinical confounders. We studied whether clinical confounders to the neurological examination modified CT-angiography diagnostic accuracy. Methods: Systematic review and meta-analysis of studies including deeply comatose patients undergoing DNC ancillary testing. We estimated pooled sensitivities and specificities using a Bayesian hierarchical model, including data on CT-angiography (4-point, 7-point, 10-point scales, and no intracranial flow), and performing a subgroup analysis on clinical confounders to the reference neurological examination. Results: Of 40 studies included in the meta-analysis, 7 involve CT-angiography $(n=586)$. There was no difference between subgroups (Table). The degree of uncertainty involving sensitivity estimates was high in both subgroups. Conclusions: Statistical uncertainty in diagnostic accuracy estimates preclude any conclusion regarding the impact of clinical confounders on CT-angiography diagnostic accuracy. Further research is required to validate CT-angiography as an accurate ancillary test for DNC.

Table. Pooled sensitivities and specificities of CT-angiography for death by neurological criteria

\begin{tabular}{|c|c|c|}
\hline $\begin{array}{l}\text { Ancillary test (radiological criteria) } \\
\text { [number of patients pooled] }\end{array}$ & $\begin{array}{c}\text { Pooled sensitivity } \\
(95 \% \text { highest } \\
\text { density interval) }\end{array}$ & $\begin{array}{c}\text { Pooled specificity } \\
(95 \% \text { highest } \\
\text { density interval) }\end{array}$ \\
\hline CT-angiography (4-point scale) $[\mathrm{N}=303]$ & $0.81(0.57-0.94)$ & $1.00(1.00-1.00)$ \\
\hline Clinical confounders $(n=197)$ & $0.82(0.62-0.93)$ & $1.00(1.00-1.00)$ \\
\hline No clinical confounders $(n=106)$ & $0.78(0.25-0.97)$ & $1.00(1.00-1.00)$ \\
\hline CT-angiography (7-point scale) $[\mathrm{N}=79]$ & $0.93(0.63-0.99)$ & $1.00(0.99-1.00)$ \\
\hline Clinical confounders $(n=79)$ & $0.90(0.64-0.99)$ & $1.00(0.99-1.00)$ \\
\hline No clinical confounders $(n=0)$ & $0.95(0.27-1.00)$ & $1.00(0.99-1.00)$ \\
\hline CT-angiography (10-point scale) $[\mathrm{N}=54]$ & $0.87(0.34-0.99)$ & $1.00(0.99-1.00)$ \\
\hline Clinical confounders $(n=54)$ & $0.84(0.37-0.98)$ & $1.00(1.00-1.00)$ \\
\hline No clinical confounders $(n=0)$ & $0.90(0.03-0.98)$ & $1.00(0.99-1.00)$ \\
\hline $\begin{array}{l}\text { CT-angiography (no intracranial flow) } \\
{[\mathrm{N}=150]}\end{array}$ & $0.89(0.55-0.98)$ & $1.00(0.99-1.00)$ \\
\hline Clinical confounders $(n=70)$ & $0.90(0.65-0.98)$ & $1.00(1.00-1.00)$ \\
\hline No clinical confounders $(n=80)$ & $0.93(0.40-1.00)$ & $1.00(0.99-1.00)$ \\
\hline
\end{tabular}

